\title{
ON A NONLINEAR INVERSE PROBLEM IN VISCOELASTICITY
}

\author{
H.D. Bui ${ }^{1}$ and S. Chaillat ${ }^{2}$ \\ ${ }^{1}$ Ecole Polytechnique/LMS, Palaiseau and Electricite de \\ France/RD/LaMSID, Clamart, France \\ ${ }^{2}$ Georgia Institute of Technology/College of Computing, USA
}

\begin{abstract}
We consider an inverse problem for determining an inhomogeneity in a viscoelastic body of the Zener type, using Cauchy boundary data, under cyclic loads at low frequency. We show that the inverse problem reduces to the one for the Helmholtz equation and to the same nonlinear Calderon equation given for the harmonic case. A method of solution is proposed which consists in two steps : solution of a source inverse problem, then solution of a linear Volterra integral equation.
\end{abstract}

\section{INTRODUCTION}

Inverse problems for defect and crack identification in elasticity and viscoelasticity have many applications in medecine and the mechanics of materials. In medecine, tomography techniques using mechanical loads such as antiplane shear loading on life tissue, considered as a viscoelastic medium, have been worked out for Kelvin-Voigt's viscoelasticity (Catheline et al [12], Muller et al [18]) and for pure elasticity. In the elastic case, solutions to crack inverse problems in 2D and 3D are already known, see Andrieux and Ben Abda [4], Andrieux et al [5], Bui et al [8]. It is thus important to know if viscoelastic inverse problems can be studied by using classical correspondence between viscoelasticity and elasticity. Such a correspondence allows for applications for mechanical tomography in viscoelastic media. We shall consider a more general viscoelastic constitutive equation of the Zener type and consider either dynamic antiplane shear loading or scalar acoustic problems in viscous materials. It has been established in Chaillat and Bui [13] that, for low frequency of the load, the correspondence between viscoelasticity and elasticity still exists. Therefore, the inverse problem for crack and defect identification in viscoelasticity can be solved by using methods available in linear elasticity. For example, the crack inverse problem in dynamic viscoelasticity can be studied by considering the same problem in the frequency domain for the scalar Helmholtz equation, which has been solved by Alves and Ha-Duong [1], [2], Ben Abda et al [6] or in the time domain by using solutions to planar crack inverse problems in transient acoustics and elastodynamics [9]. In this paper we shall consider the inverse problem for identifying a distributed defect in viscoelasticity with potential applications to detection of tumors in soft tissue and show that the perturbation of the material constant $\mathrm{h}(\mathrm{x})$ satisfies the same nonlinear equation 
established by Calderon [11] for the quasistatic case. While the linearized equation, for infinitely small perturbation, has been solved analytically by Calderon [11], the nonlinear case can be studied by considering two problems, a source inverse problem and a Volterra integral equation of the first kind.

\section{A VISCO-ELASTIC MODEL}

\subsection{Stress-strain law}

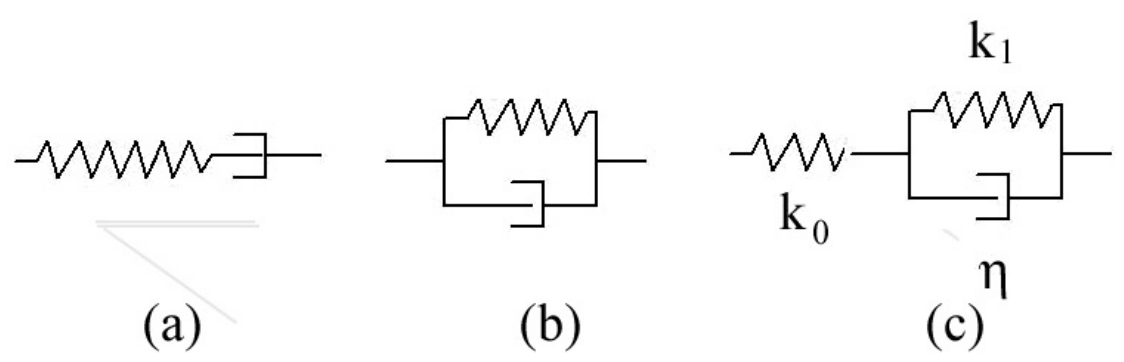

Fig. 1. Viscoelastic models: (a) Maxwell; (b) Kelvin-Voigt; (c) Zener

Various rheological models exist to model the viscoelastic behavior of a material. The Kelvin-Voigt model is well suitable to model solids. The Maxwell model is generally used to model fluids. The rheological model used in this paper is a Zener one with a dashpot impedance $\eta$ and elastic constants $k_{0}$ and $k_{1}$ (Fig. 1c). In this model, when $\dot{\varepsilon}=0$, the dashpot has no effect and the model has a "delayed modulus". The model is equivalent to two springs connected in series. On the other hand, if $\dot{\varepsilon}=\infty$, the dashpot is blocked, it has an instantaneous modulus. Usual formulation of viscoelasticity is given by the Boltzmann functional equation (Mandel [16])

$$
\sigma_{i j}(t)=\int_{0}^{t} \lambda(t-\tau) \delta_{i j} \dot{\varepsilon}_{k k}(\tau) d \tau+\int_{0}^{t} 2 \mu(t-\tau) \dot{\varepsilon}_{i j}(\tau) d \tau
$$

with $\lambda(\mathrm{t})$ and $\mu(\mathrm{t})$ being relaxation functions. Another constitutive law is given by the differential form

$$
\frac{1}{\eta} \boldsymbol{\sigma}+\dot{\boldsymbol{\sigma}}=\Lambda_{\infty}:\left(\frac{1}{\gamma} \varepsilon+\dot{\varepsilon}\right)
$$

where $\Lambda_{\infty}=\lambda_{\infty} I_{2} \otimes I_{2}+2 \mu_{\infty} I_{4}$ is the instantaneous elastic moduli tensor, defined by instantaneous Lamé's constants $\lambda_{\infty}, \mu_{\infty}$ and $\gamma, \eta$ are respectively creep and relaxation times. In what follows, we change notations and rewrite (2) in the form proposed by Goriacheva [14] with the delayed elastic tensor $\Lambda$ defined with Lamé's constants $\lambda, \mu$

$$
\boldsymbol{\sigma}+\beta \dot{\boldsymbol{\sigma}}=\Lambda:(\varepsilon+\alpha \dot{\boldsymbol{\varepsilon}})
$$

The coefficients $\alpha, \beta$ are determined by $\alpha=\eta / k_{1}, \beta=\eta /\left(k_{0}+k_{1}\right)$, Fig. (1). Remark that the material in the interface between two sliding solids, which behaves like an elastic solid 
in compression and a viscous fluid in tangential, can be described by (3), see DragonLouiset [17]. We introduce the transformed stress, displacement and strain

$$
\begin{gathered}
\boldsymbol{\sigma}^{*}=\boldsymbol{\sigma}+\beta \frac{\partial \boldsymbol{\sigma}}{\partial t} \\
\boldsymbol{u}^{*}=\boldsymbol{u}+\alpha \frac{\partial \boldsymbol{u}}{\partial t}, \quad \boldsymbol{\varepsilon}^{*}=\boldsymbol{\varepsilon}+\alpha \frac{\partial \boldsymbol{\varepsilon}}{\partial t}
\end{gathered}
$$

The tensors $\boldsymbol{\sigma}^{*}$ and $\varepsilon^{*}$ are linked by the constitutive equation of isotropic elasticity with the Lamé coefficients $\lambda$ and $\mu$ of the delayed moduli

$$
\sigma^{*}=\Lambda: \varepsilon^{*}
$$

With $\boldsymbol{u}(\boldsymbol{x}, t)=\boldsymbol{v}(\boldsymbol{x}) \cos (\omega t)$, the displacement rate $\dot{\boldsymbol{u}}(\boldsymbol{x}, t)$ is out of phase of $\pi / 2$. Using (5), we obtain $\boldsymbol{u}^{*}(\boldsymbol{x}, t)=\boldsymbol{v}(\boldsymbol{x})(\cos (\omega t)-\alpha \omega \sin (\omega t))$.

Defining the angle $\psi$ such that $\tan \psi=\alpha \omega(0 \leq \psi<\pi / 2$, i.e. $\cos \psi \neq 0)$ it follows:

$$
\boldsymbol{u}^{*}(\boldsymbol{x}, t)=\frac{\boldsymbol{v}(\boldsymbol{x})}{\cos (\psi)} \cos (\omega t+\psi)
$$

It is now clear that variables $\boldsymbol{u}^{*}$ and $\boldsymbol{u}$ are out of phase of angle $\psi$ but have the same circular frequency $\omega$. Then, suppose that $\boldsymbol{\sigma}(\boldsymbol{x}, t)=\boldsymbol{w}(\boldsymbol{x}) \cos (\omega t+\theta)$, we obtain $\boldsymbol{\sigma}^{*}(\boldsymbol{x}, t)=\boldsymbol{w}(\boldsymbol{x})[\cos (\omega t+\theta)-\beta \omega \sin (\omega t+\theta)]$. Defining in the same manner the angle $\phi$ such that $\tan \phi=\beta \omega(0 \leq \phi<\pi / 2)$ it follows:

$$
\boldsymbol{\sigma}^{*}(\boldsymbol{x}, t)=\frac{\boldsymbol{w}(\boldsymbol{x})}{\cos (\phi)} \cos (\omega t+\phi+\theta)
$$

The variables $\boldsymbol{\sigma}^{*}$ and $\boldsymbol{u}^{*}$ are known to satisfy the constitutive equation (6), so that they have to be in phase. It follows that $\psi=\phi+\theta$. Finally, stress $\boldsymbol{\sigma}$ and strain or $\boldsymbol{u}$ have to be out of phase of $\theta=\psi-\phi$.

\subsection{Equation of motion}

The quantities $\boldsymbol{\sigma}^{*}$ and $\boldsymbol{u}^{*}$ which are linked by elastic law, are now shown to satisfy the equation of motion approximately. The assumed form of fields $\boldsymbol{\sigma}$ and $\boldsymbol{u}$ are compatible with the equation of motion $\operatorname{div} \boldsymbol{\sigma}-\rho \ddot{\boldsymbol{u}}=0$ if and only if the out of phase angle $\theta$ is very small. More precisely, the modification introduced in the dynamic equation by the phase difference between $\boldsymbol{\sigma}$ and $\boldsymbol{u}$ is proportional to $\rho \omega^{2} \theta$. Since for small $\theta$, we have $\theta \approx|\alpha-\beta| \omega$ and thus $\rho \omega^{2} \theta \approx \rho \omega^{3}|\alpha-\beta|$, where $\alpha=\eta / k_{1}, \beta=\eta /\left(k_{0}+k_{1}\right)$, using $\boldsymbol{u}(\boldsymbol{x}, t)=\boldsymbol{v}(\boldsymbol{x}) \cos \omega t$. It follows that

$$
\operatorname{div} \boldsymbol{\sigma}^{*}-\rho \ddot{\boldsymbol{u}^{*}}=\rho(\beta-\alpha) \partial_{t} \ddot{\boldsymbol{u}} \approx \rho \omega^{3}|\alpha-\beta|\|\boldsymbol{v}\|
$$

The latter term can be neglected in comparison with $\rho \omega^{2}\|\boldsymbol{v}\|$ if and only if

$$
\theta \approx|\alpha-\beta| \omega \ll 1
$$

Therefore, the frequency of the experiment must be much less than the limit frequency $\omega_{1}=1 /|\alpha-\beta|$. The static case $\omega=0$ obsviously satisfies the latter condition. Since $|\alpha-\beta|$ is proportional to the viscosity coefficient $\eta$, the lower the coefficient $\eta$, the higher the limit frequency $\omega_{1}$ (in solid mechanics $\eta$ is indeed small, so that $\omega_{1}$ is large). Also $\omega_{1}$ 
is large when $|\alpha-\beta|$ is small for some rheological models. Very often, Civil Engineering problems do not consider viscosity.

Finally, provided that $\omega \ll \omega_{1}$, the change of functions using $\boldsymbol{u}^{*}$ and $\boldsymbol{\sigma}^{*}$ leads to the same elastodynamic equation $\operatorname{div} \boldsymbol{\sigma}^{*}+\rho \omega^{2} \boldsymbol{u}^{*}=0, \boldsymbol{\sigma}^{*}=\Lambda: \boldsymbol{\varepsilon}^{*}$. This correspondence between viscoelasticity and elasticity making use of a real Helmholtz equation has been exploited in [13] for a new approach of real boundary integral equation. A crack inverse problem in viscoelasticity is considered in Bui et al [10]. In what follows, we will exploit some known methods of inverse problems for elasticity and for the Helmholtz equation in order to study the change of constant, for example due to damage in a material or to tumor growth in life tissue.

\section{THE NONLINEAR CALDERON EQUATION}

In what follows we consider the identification of internal defects in 2D problems. For example, an antiplane shear loading of a body whose normalized shear modulus is of the form $m(\boldsymbol{x})=1+h(\boldsymbol{x})$. The shear modulus in the absence of defect $(h=0)$ is normalized to unity. For scalar acoustics, the bulk modulus is $m(\boldsymbol{x})=1+h(\boldsymbol{x})$. We assume that the defect is characterized by function $h(\boldsymbol{x})$ with compact support $C \subset \Omega$ and $h=0$ on the boundary $\partial C$. We extend its definition outside the inclusion by setting $h=0$ there. The governing equations are $\operatorname{div}(m \operatorname{grad} u)+k^{2} u=0$, with prescribed $(1+h) \partial u / \partial n=\partial u / \partial n=\mathrm{w}$ on the boundary. The boundary value $u=\mathrm{v}$ can be measured for further use as a data in the inverse problem. In anti-plane shear loading of a long cylinder, $u\left(x_{1}, x_{2}\right)$ is the out of plane displacement component (along the axis of the cylinder), while in acoustics $u\left(x_{1}, x_{2}\right)$ denotes the pressure. Using the transformed function $u^{*}$ for small $k^{2}$, the equations are

$$
\begin{gathered}
\operatorname{div}\left\{(1+h(\boldsymbol{x})) \operatorname{grad} u^{*}\right\}+k^{2} u^{*}=0 \text { in } \Omega \\
u^{*}=f \equiv v / \cos \psi, \quad \frac{\partial u^{*}}{\partial n}=g \equiv w / \cos \phi \quad \text { on } \partial \Omega
\end{gathered}
$$

These equations are precisely the same as the ones considered in the Helmholtz inverse problem. For $k=0$, the linearized inverse problem for small perturbation $h$ (Fig. 2), has been solved by Calderon [11], see also [7]. Let us generalize Calderon's problem to Eqs. (10-11) for $k \neq 0$.

Condider an adjoint problem for the sound solid $h=0$ :

$$
\begin{gathered}
\text { div } \operatorname{grad} \varphi+k^{2} \varphi=0 \text { in } \Omega, \\
\frac{\partial \varphi}{\partial n}=a(\boldsymbol{x}) \text { on } \partial \Omega
\end{gathered}
$$

and let $b(\boldsymbol{x})$ be the boundary value of $\varphi$.

Combining (10) and (12a), making an integration by parts and taking account of $h=0$ on $\partial C$, we get an equation similiar to the one given in Calderon [11]

$$
\int_{C} h(\boldsymbol{x}) \operatorname{grad} u^{*}(\boldsymbol{x} ; h) \cdot \operatorname{grad} \varphi(\boldsymbol{x}) d^{2} x=\int_{\partial \Omega}(g b-f a) d S:=R(f, g ; a, b)
$$




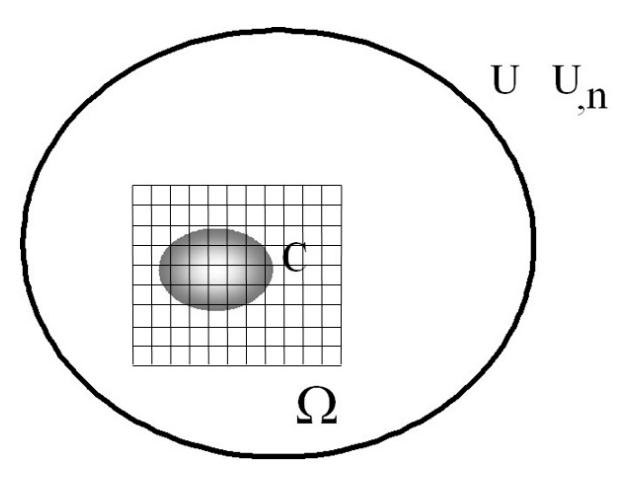

Fig. 2. Identification of an inclusion $\mathrm{C}$ represented by a perturbation of the material constant $\lambda$

The right hand side of Eq. (13) is known from the boundary data $(f, g)$ of the current field $u^{*}$ and the data $(a, b)$ of the adjoint function $\varphi$. In the following, it will be denoted by $R(f, g ; a, b)$. Eq. (13) is nonlinear in h and its integration domain $C$ is yet unknown.

Following Calderon [11] we can linearize Eq. (13) by replacing the unknown function $u^{*}$ by function $u^{0}$ which satisfies

We obtain

$$
\operatorname{div} \operatorname{grad} u^{0}+k^{2} u^{0}=0 \quad \text { in } \Omega, \quad \frac{\partial u^{0}}{\partial n}=g \text { on } \partial \Omega
$$

$$
\int_{C} h(\boldsymbol{x}) \operatorname{grad} u^{0}(\boldsymbol{x}) \cdot \operatorname{grad} \varphi(\boldsymbol{x}) d^{2} x=R(f, g ; a, b)
$$

An explicit solution of Eq. (15) can be derived by using adjoint field of the Helmholtz equation and similar method outlined by Calderon [11].

\subsection{A source inverse problem}

We need some preliminary results on the source inverse problem which consists in finding the source $S(\boldsymbol{x})$ using Cauchy boundary data $f$ and $g$

$$
\begin{gathered}
\text { div } \operatorname{grad} u+k^{2} u+S(\boldsymbol{x})=0 \text { in } \Omega \\
u=f \text { on } \partial \Omega, \quad \frac{\partial u}{\partial n}=g \text { on } \partial \Omega
\end{gathered}
$$

This problem has been investigated in the literature, for the harmonic equation $(k=0)$ as well for the Helmholtz equation, see Isakov [15], El Badia and Ha-Duong [1]. The source inverse problem does not generally have a unique solution. For example, in potential theory $(k=0)$ two sources of the form $S_{i}=\lambda_{i} \chi\left[\omega_{i}, \boldsymbol{a}_{i}, r_{i}\right], i=1,2$ with characteristic functions $\chi$ of balls $\omega_{i}$, center $\boldsymbol{a}_{i}$, radius $r_{i}$, amplitude $\lambda_{i}$, give the same pair of boundary data $f, g$ when they have the same centers $\boldsymbol{a}_{1}=\boldsymbol{a}_{2}$ and $\lambda_{1} r_{1}^{2}=\lambda_{2} r_{2}^{2}$, Isakov [15], El Badia and Ha-Duong [1]. To ensure uniqueness [19], one considers the class $V$ of sources defined by the finite sum $\sum \lambda_{i} \chi\left[\omega_{i}, \boldsymbol{a}_{i}, r_{i}\right]$, with distinct centers, as proposed in [1]. Upon discretizing the expected source zone into M square elements (or pixels) $s_{i}$ of size $e_{i}$, with meshes lying entirely inside the domain (Fig. 2), any function $\mathrm{S}(\mathrm{x})$ can be approximated 
by a finite sum of piecewise constant functions in distinct square supports, with constant intensities $S(x)=\lambda_{i}^{\prime} \chi\left[s_{i}, e_{i}\right]$. Function $S(\boldsymbol{x})$ is well approximated by this way. However, to ensure uniqueness of results it is convenient to use a basis of circular sources discussed above $\lambda_{i} \chi\left[\omega_{i}, \boldsymbol{a}_{i}, r_{i}\right]$. Many choices are possible : inscribed circle with radius $e_{i} / 2$ with intensity $\lambda_{i}=4 \lambda_{i}^{\prime} / \pi$, circular source with the same area or $r_{i}=e_{i} / \sqrt{\pi}$. The circular shape is important because, as remarked in [1], from the boundary observation, i.e the functional $\mathrm{R}$, sources in $V$ behaves like point sources. Given a source domain $C$, the question is how much circular sources $\omega_{i}$ of supports in $C$ and of distinct centers and different radii may be considered for approximation? A large number of elementary sources gives a better approximation of $\mathrm{C}$, in the sense that the measure of $C \backslash \bigcup \omega_{i}$ is small, but the corresponding linear system may be ill-posed. A small number of elementary sources allows fair invertibility of the linear system but provides poor approximation of $C$. The best choice is an open problem. In the following, we assume that Cauchy data corresponds to some unknown source of the form $S(x)=\sum_{i} \lambda_{i}^{\prime} \chi\left[s_{i}, e\right]$. For any field $\varphi$ solution in the set $A$ of solution which satisfies either the harmonic equation $(k=0)$, or the homogeneous Helmholtz equation $(k \neq 0)$, we get

$$
\int_{\partial \Omega}\left(\varphi \frac{\partial u}{\partial n}-u \frac{\partial \varphi}{\partial n}\right) d s+\int_{C} \varphi S(\boldsymbol{x}) d^{2} x=0, \quad \forall \varphi \in A
$$

and using the definition of $R$ we can write

$$
R(\varphi)+\int_{C} \varphi S(\boldsymbol{x}) d^{2} x=0, \quad \forall \varphi \in A
$$

Equations (18) or (19) show that two non zero sources $S_{i}(\boldsymbol{x}) \neq 0$ of distinct supports in the class $\mathrm{V}$, giving the same boundary data $\mathrm{f}$, g cannot exist, because by difference, we have $\int_{\Omega} \varphi(\boldsymbol{x})\left(S_{1}(\boldsymbol{x})-S_{2}(\boldsymbol{x})\right) d^{2} x=R(2)-R(1)=0$, for any $\varphi$ in $A$. The conclusion is that these sources vanish separately (because of distinct supports) in contradiction with our assumption. Equation (19) allows the construction of solution $S(\boldsymbol{x})$ by considering a family of $\mathrm{M}$ adjoint fields $\varphi^{(j)}, j=1, . . M$ such that the matrix of the following linear system of equations for $\lambda_{i}$ is invertible

$$
R\left(\varphi^{(j)}\right)+e^{2} \sum_{i=1}^{M} \lambda_{i} \varphi\left(\boldsymbol{a}_{i}\right)^{(j)}=0, \quad j=1, . . M
$$

For $k=0$, harmonic adjoint fields are the real (or imaginary) part of polynomial of $z=x_{1}+i x_{2}$,

$$
\varphi(\boldsymbol{x})^{(j)}=\Re\left(z^{j-1}\right), \quad j=1, . . M
$$

The matrix in $(20) A_{i j}=\varphi\left(\boldsymbol{a}_{i}\right)^{(j)}$ of the Vandermonde type is invertible. Generally the number $M$ is not known. One assumes that there exists a maximun number $M_{\max }$ (a priori knowledge). The linear system (20) has the rank $M<M_{\max }$ which may be determined by studing eigenvalues of the matrix. For $k \neq 0$, there are many possible adjoint fields. The first one, for Eq.(20), is given by the real part of function, Ammari and 
Ramm [3]

$$
\varphi\left(\boldsymbol{x}, \boldsymbol{\xi}^{(j)}\right)=\exp \left[-i \boldsymbol{x} \cdot\left(\boldsymbol{\xi}^{(j)}+i \gamma \boldsymbol{\xi}^{\perp(j)}\right)\right] \quad j=1, . . M
$$

where $\boldsymbol{\xi}^{\perp(j)}=\boldsymbol{e}^{3} \times \boldsymbol{\xi}^{(j)}, \gamma=\left(1 /\left\|\boldsymbol{\xi}^{(j)}\right\|\right) \sqrt{\boldsymbol{\xi}^{(j) 2}-4 k^{2}} \quad$ if $\left\|\boldsymbol{\xi}^{(j)}\right\|>2 k, \quad$ and $\gamma=-i\left(1 /\left\|\boldsymbol{\xi}^{(j)}\right\|\right) \sqrt{4 k^{2}-\boldsymbol{\xi}^{(j) 2}}$ if $\left\|\boldsymbol{\xi}^{(j)}\right\|<2 k$.

The second one is the $\xi$-family of $2 \mathrm{D}$ fundamental solution of the Helmholtz equation with singular point $\boldsymbol{\xi}$ lying outside the domain

$$
\varphi(\boldsymbol{x}, \boldsymbol{\xi})=\frac{i}{4} H_{0}^{1}(k\|\boldsymbol{x}-\boldsymbol{\xi}\|), \quad \boldsymbol{\xi} \notin \Omega, \quad \boldsymbol{x} \in \Omega
$$

with Hankel function of the 1rst kind and order 0 . One chooses M different singular points $\boldsymbol{\xi}^{(j)}$ outside the domain and near its boundary.

\subsection{Reduction to two successive simpler problems}

Let us rewrite Eq.(13) in the following form

$$
\operatorname{div} \operatorname{grad} u^{*}+k^{2} u^{*}+\underbrace{\operatorname{div}\left(h(\boldsymbol{x}) \operatorname{grad} u^{*}(\boldsymbol{x} ; h)\right)}_{\mathrm{H}(\boldsymbol{x})}=0 \text { in } \Omega
$$

where the underbraced term is denoted by $H(\boldsymbol{x})$. The equation

$$
\operatorname{div} \operatorname{grad} u^{*}+k^{2} u^{*}+H(\boldsymbol{x})=0 \text { in } \Omega
$$

with unknown $H(\boldsymbol{x})$ and boundary data $u^{*}=f$ and $\partial u^{*} / \partial n=g$ is a source inverse problem. By solving this source inverse problem (see Subsection 3.1) we obtain the source $H(\boldsymbol{x})$, its support $C$ and the true displacement field $U(\boldsymbol{x}) \doteq u^{*}(\boldsymbol{x} ; h)$, even if $h(\boldsymbol{x})$ is unknown. We obtain also the strain, but not the stress.

It is interesting to remark that the solution of the source inverse problem (25) provides the support of the unknown $h(\boldsymbol{x})$, since it can be proved that $\operatorname{supp}(H) \equiv \operatorname{supp}(h)=C$. It does not provide the function $h$ itself. Hereafter, we assume that the support $C$ of $H$ is known. To determine $h$, we remark that the term $u^{*}(\boldsymbol{x} ; h)$ of the nonlinear Calderon equation (13) can be replaced by the field $U(\boldsymbol{x})$ determined above, thus

$$
\int_{C} h(\boldsymbol{x}) \operatorname{grad} U(\boldsymbol{x}) \cdot \operatorname{grad} \varphi(\boldsymbol{x}) d^{2} x=R(\varphi)
$$

Next we introduce the $\xi$-family of adjoint field $\varphi(\boldsymbol{x}, \boldsymbol{\xi})$ defined by the fundamental solution of the Helmholtz equation (23) where the singular point is the parameter $\boldsymbol{\xi} \in$ $\mathbb{R} \backslash \Omega$. We obtain a Volterra integral equation of the first kind, with kernel $K(\boldsymbol{x}, \boldsymbol{\xi}) \doteq$ $\operatorname{grad} U(\boldsymbol{x}) \cdot \operatorname{grad} \varphi(\boldsymbol{x}, \boldsymbol{\xi})$

$$
\int_{C} h(\boldsymbol{x}) K(\boldsymbol{x}, \boldsymbol{\xi}) d^{2} x=\int_{\partial \Omega}\left(\varphi \frac{\partial u}{\partial n}-u \frac{\partial \varphi}{\partial n}\right) d s \doteq R(\varphi, \boldsymbol{\xi})
$$

The nonlinear Calderon problem is solved if we find $h(\boldsymbol{x})$ satisfying $h=0$ on $\partial C$ and the above linear Volterra integral equation of the first kind, over a known domain $C$. 


\section{CONCLUSIONS}

In this paper we consider a particular viscoelastic stress-strain law involving stress and strain rates of the Zener type. Provided that the frequency is low, the correspondence between viscoelasticity and elasticity is conserved. Some inverse problems for defect identification in viscoelasticity, with applications to medical tomography, can be solved by existing corresponding solutions in elasticity or scalar acoustics. We have shown that the nonlinear Calderon problem reduces to two successive problems: a source inverse problem and a Volterra integral equation of the first kind.

\section{REFERENCES}

[1] C.J.S. Alves and T. Ha-Duong. An inverse source problem in potential analysis. Inverse Problems 23 (2000) 651-666.

[2] C.J.S. Alves and T. Ha-Duong. On inverse scattering by screen. Inverse Problems 13 (1997) 1161-1176.

[3] H. Ammari and A. G. Ramm. Recovery of small electromagnetic inhomogeneities from partial boundary measurements. C. R. Mecanique 330 (2002) 199-205.

[4] S. Andrieux. and A. Ben Abda. Identification de fissures planes par une donnee de bord unique: un procede direct de location et d'identification. C.R. Acad. Sci. Paris, 315 (I) (1992), 13231328.

[5] S. Andrieux, A. Ben Abda and H.D. Bui. Reciprocity principle and crack identification. Inverse Problems 15 (1999) 59-65.

[6] A. Ben Abda, F. Delbary and H. Haddar. On the use of the reciprocity-gap functional in inverse scattering from planar cracks. Mathematical Models and Methods in Appl. Sci. 115 (2005) 1553-1574.

[7] H.D. Bui. Bai toan nguoc trong co hoc vat lieu, Nguyen Dong Anh (Ed). NXBXD Ha Noi, 1996. Translated from Inverse problems in the mechanics of materials : an introduction CRC Press, Boca Raton 1994.

[8] H.D. Bui, A. Constantinescu and H.Maigre. An exact inversion formula for determining a planar fault from boundary measurement. J. Inv. Ill-Posed Problems 13, (2005) 553-565.

[9] H.D. Bui. Fracture Mechanics. Inverse problems and solution, Springer, 2006.

[10] H.D. Bui, S. Chaillat, A. Constantinescu and E. Grasso. Identification of defects in viscoelasticity, (Submitted) 2009.

[11] A. P. Calderon. On an inverse boundary problem. In Seminar on numerical and applications to continuum physics. W.H. Meyer and M. A. Raupp (Eds.) Brazilian Math. Soc. Rio de Janerio, (1980) 65-73.

[12] S. Catheline, J.L. Gennisson, G. Delon, M. Fink, R. Sinkus, S. Abouelkaram, J. Culioli. Measuring of viscoelastic properties of homogeneous soft solid using transient elastography: an inverse problem approach. J. Acoust. Soc. Am. 116(6), (2004) 3734-3741.

[13] S. Chaillat and H.D. Bui. Resolution of linear viscoelastic equations in the frequency domain using real Helmholtz boundary integral equations. Comptes Rendus de Mecanique 335 (2007) 746-750.

[14] I.G. Goriacheva. Contact problem of rolling of a viscoelastic cylinder on a base of the same material. PMM 37 (1973) 925-933.

[15] V. Isakov. Inverse sources problems. Math. Surveys and Monographs 34(1990) Providence RI, Am. Math. Soc.

[16] J. Mandel. Viscoelasticite C.R. Acad. Sci.241 (1955) 1910. 
[17] M. Dragon-Louiset. On a predictive macroscopic contact-sliding wear model based on micromechanical considerations. Int. J. Solids and Structures 38 (2001) 1625-1639.

[18] M. Muller, J.L. Genisson, T. Deffieux, R. Sinkus, A. Philippe, G. Montaldo, M. Tanter and M. Fink. Full 3D inversion of the viscoelasticity wave propagation problem for 3D Ultrasound elastography in Breast Cencer Diagnosis. Ultrasonics Symposium IEEE (2007) 672-675.

[19] L. Rondi. Uniqueness for the determination of sound soft defects in an inhomogeneous planar medium by acoustic boundary measurements. Trans. Am. Math. Soc. 38 (2002) 321-239.

Received June 26, 2009

\section{GIẢI BÀI TOÁN NGƯợC ĐÀN NHỚT PHI TUYẾN}

Chúng ta xem xét một bài toán ngược để xác định tính không thuần nhất trong vật thể đàn nhớt Zener, sử dụng dữ liệu biên Cauchy, dưới tải tuần hoàn ở tần số thấp. Chúng ta thấy rằng bài toán ngược rút gọn thành một phương trình Helmholtz và phương trình phi tuyến Calderon lấy cho trường hợp điều hòa. Một phương pháp để tìm nghiệm của bài toán được đưa ra bao gồm hai bước: nghiệm của bài toán ngược gốc, sau đó nghiệm của phương trình tích phân Volterra tuyến tính. 